\title{
Ganciclovir Resistant Cytomegalovirus in Solid Organ Transplant Recipients: An Update
}

\author{
Chandandeep Takkar, Ann K Gamilla-Crudo and Pradeep V Kadambi*
}

Division of Nephrology and Hypertension, The University of Texas Medical Branch, USA

\begin{abstract}
Ganciclovir-resistant cytomegalovirus (CMV) infection is an emerging problem in solid organ transplant (SOT) recipients. Treatments such as foscarnet and cidofovir are fraught with serious side effects that may limit their use in this condition. The aim of this paper is to elucidate the mechanisms on how resistance occurs, when to suspect it clinically and what special tests are necessary to diagnose it. Based on recent literature, the paper also brings to light two medications, maribavir and leflunomide, which have been described to have anti-CMV activity.
\end{abstract}

Keywords: Ganciclovir resistance; Cytomegalovirus; Leflunomide; Kidney transplantation; Solid organ transplant

Abbreviations: CMV: Cytomegalovirus; GCV: Ganciclovir; SOT: Solid Organ Transplant; MBV: Maribavir

\section{Introduction}

Antiviral resistance in CMV poses an important therapeutic challenge in SOT recipients. Ganciclovir (GCV) is the current mainstay of treatment for CMV infections. We summarize the current understanding of epidemiology, pathogenesis, clinical manifestations, diagnosis and management strategies for GCV resistant CMV infections in SOT recipients, particularly kidney transplants.

While the precise incidence of GCV resistance is undefined, it may affect up to $7 \%$ of SOT recipients, with highest risk among kidneypancreas and lung transplant patients [1-15].

\section{Mechanisms of Resistance}

In order to understand the mechanisms of resistance to antivirals in CMV, it is important to review the mode of action of GCV. It is a guanosine analogue that must be converted into its triphosphorylated form to be active. Once triphosphorylated, it inhibits viral DNA synthesis by inhibition of CMV DNA polymerase (encoded by viral gene, UL54). After cell entry, the phosphorylation of GCV occurs in three sequential steps, first of which requires a virally encoded phosphotransferase, a product of UL97. The following phosphorylation steps are performed by host cellular enzymes (Figure 1). Mutations in UL97 leading to reduced levels of active form of GCV, and those in UL54 leading to CMV polymerase resistance to GCV, are the major mechanisms of resistance.

Many of the common mutations in UL97 and UL54 correlate with phenotypic resistance to GCV by in vitro studies [16]. The correlation between genotypic mutations, phenotypic resistance and clinical refractoriness to GCV has formed the basis for the development of genotypic screens for resistance [17]. Furthermore, it appears that UL97 region mutations are more common than those of UL54. However, UL54 mutations are typically associated with higher levels of resistance to GCV and cross resistance to cidofovir and foscarnet $[18,19]$.

\section{Risk Factors}

New understanding into the emergence of resistant CMV strains has developed after studies were done by Emery et al. [20,21]. They have shown that in the presence of GCV, mutant CMV strains have a survival advantage compared to wild type CMV. During prolonged exposure to GCV, especially when the systemic drug levels are low (oral GCV or valganciclovir), these mutant strains may become the dominant population leading to treatment failure. This information helps understand several clinical observations. For instance, antiviral resistance is associated with high viral titers since there is a greater opportunity for selection of resistant mutant strains. Furthermore, resistance is usually encountered after prolonged periods of exposure to GCV, presumably because there is a growth advantage for mutant strains in the presence of the drug. Lastly, GCV resistance develops more commonly after oral rather than intravenous administration. This is because the blood levels achieved with oral GCV are much lower than those obtained after intravenous therapy, leading to incomplete suppression of viral replication.

Risk factors include a donor seropositive and recipient seronegative (D+/R-) status for CMV, prolonged GCV exposure, potent immunosuppression and high viral titers [22]. The observation that $\mathrm{D}+/$ $\mathrm{R}$ - status almost seems necessary (except in lung transplant recipients) in the development of CMV resistance narrows the population at risk. This predisposition is likely due to lack of previous immunity to CMV.

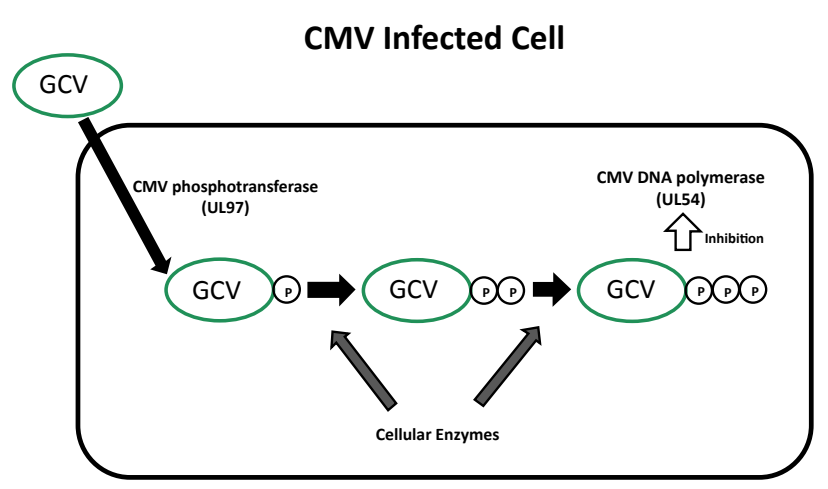

Figure 1: Mechanism of action of Ganciclovir in CMV infection.

*Corresponding author: Pradeep V Kadambi, Division of Nephrology and Hypertension, The University of Texas Medical Branch, 301, University Blvd, 4.200 JSA, Galveston, TX 77555, USA, Tel: 1-409-772-1811; Fax: 1-409-772-5451; E-mail: pradeep.kadambi@utmb.edu

Received March 29, 2013; Accepted May 24, 2012; Published May 27, 2012

Citation: Takkar C, Gamilla-Crudo AK, Kadambi PV (2013) Ganciclovir Resistant Cytomegalovirus in Solid Organ Transplant Recipients: An Update. J Transplant Technol Res S6: 001. doi:10.4172/2161-0991.S6-001

Copyright: (C) 2013 Takkar C, et al. This is an open-access article distributed under the terms of the Creative Commons Attribution License, which permits unrestricted use, distribution, and reproduction in any medium, provided the original author and source are credited. 
Lung transplantation seems to be an exception to this, since antiviral resistant CMV has also been noted in $\mathrm{R}+$ setting [22]. It is possible that these patients have other issues that are conducive to development of CMV resistance.

\section{Clinical Features}

The spectrum of clinical manifestations of GCV resistant CMV can range from asymptomatic viremia to the CMV syndrome as well as tissue invasive disease. Since these strains have been demonstrated in diseased tissue [23], it could be concluded that despite reduced potential to replicate $[20,21]$, they maintain their pathogenicity. Based upon published cases of GCV resistant CMV in SOT patients, about $87 \%$ have symptomatic infection, with $55 \%$ of those having tissue invasive disease [1-15]. The mortality rate in affected patients approaches $20 \%$, although the mortality directly attributable to resistant CMV is difficult to ascertain given the anecdotal nature of most data [1-15].

Due to routine prophylaxis against CMV, the timing of CMV infection and disease has shifted later in the post transplant period compared to the era when CMV prophylaxis was not widely used [22].

Compared with other SOT patients, lung transplant patients seem to have an earlier onset of resistant CMV infection (median time post transplant 146 days vs. 279 days) [22]

\section{Diagnosis}

The diagnosis of GCV resistant CMV involves either documentation of reduced susceptibility of the CMV isolate to GCV in vitro by one of the phenotypic methods or direct identification of established genetic mutations known to correlate with resistance.

In contrast to genotypic analyses, which utilize the knowledge of genetic mutations that confer resistance to antiviral agents, phenotypic methods depend upon measuring inhibition of viral growth in the presence of varying drug concentrations compared to a control strain. Some of the phenotypic methods include measurement of viral plaques, DNA synthesis or antigen production [19]. It is proposed that on plaque reduction assay, a $50 \%$ inhibitory concentration of GCV exceeding $6 \mu \mathrm{mol} / \mathrm{L}$ be considered the threshold for diagnosis of resistant CMV [22].

Genotypic methods involve restriction enzyme analysis of polymerase chain reaction products from clinical CMV isolates and looking for known mutations associated with antiviral resistance.

One of the shortcomings of most available methods is the need for viral culture, which takes several weeks thus delaying the diagnosis. Despite recent technical advances in phenotypic and genotypic methods allowing for a quicker diagnosis [24], they are not widely available and are not standardized, thus limiting their utility in clinical decision making.

Therefore, in a high risk patient, a high index of suspicion must be maintained. Based on expert opinion, antiviral resistance should be suspected in a high risk patient when viral load fails to decline or rises after appropriate intravenous GCV administration for 14 days. Similarly, persistence of positive viral cultures or failure of clinical improvement after 14 days of intravenous GCV should suggest resistance [22]. Under such circumstances, empiric changes in antiviral regimen must be made before laboratory confirmation is available.

\section{Treatment}

Unfortunately, there are no controlled studies to guide the treatment of GCV resistant CMV specifically in the setting of SOT.
Data derived from studies in HIV population and clinical experience in SOT patients provides some guidance regarding this issue. It is important to note that not all clinical failure to therapy in CMV infection is due to resistance to antivirals. Conversely, GCV resistant infections may respond to GCV, especially when used in combination with other treatments. The degree of GCV resistance, host immune response and disease severity should all be taken into account when making therapeutic decisions. Accordingly, a multifaceted approach targeting all these factors is likely to be helpful in the management of resistant CMV infections.

Based upon genotypic studies of resistant CMV isolates, mutations in UL97 alone usually do not show cross resistance to foscarnet or cidofovir $[16,17]$. Due to the fear of nephrotoxicity with cidofovir, foscarnet is considered the first line alternative for management of resistant CMV infections. CMV hyperimmune globulin is usually added in patients with tissue invasive and severe disease. In patients who are not severely ill, an increase in GCV dose from $5 \mathrm{mg} / \mathrm{kg} / \mathrm{dose}$ to $7.5 \mathrm{mg} / \mathrm{kg} /$ dose can be utilized as well [25]. However, neutropenia and renal impairment often limit this dose increase. Mylonakis et al. showed that GCV at a dose of $5 \mathrm{mg} / \mathrm{kg}$ when combined with escalating doses of foscarnet was another useful strategy [26]. More recently, two medications have come to light in the treatment of resistant CMV disease.

Maribavir (MBV) is an investigational benzimidazole antiviral agent (1H-beta-L-ribofuranoside-2-isopropylamino-5, 6-dichlorobenzimidazole) that has in vitro activity against CMV strains that are resistant to other therapeutic agents [27]. Its effects include prevention of viral encapsidation and exit of viral particles by binding to UL97 viral protein kinase $[27,28]$. Avery et al. reported outcomes on 6 patients (5 SOT and 1 hematopoietic stem cell transplant) with treatment failure to conventional agents and/or known GCV resistance. Initially, patients received oral MBV at $400 \mathrm{mg}$ twice daily for a median duration of 207 days. Four of the six patients had no detectable viremia within six weeks of starting MBV. The authors recommended an MBV concentration of atleast $6 \mu \mathrm{g} / \mathrm{mL}$ for CMV treatment [29]. MBV was generally well tolerated with no significant renal, hepatic or hematologic toxicity.

Leflunomide, (N-(4'trifluoromethylphenyl)-5-methylisoxazole-4carboxamide) is a drug that is approved for use in rheumatoid arthritis. It not only has immunosuppressive properties but also has anti-CMV activity. Waldman and colleagues first described the use of leflunomide against CMV. Their study found that leflunomide acts through inhibition of tegument acquisition by viral nucleocapsids [30]. Evers and colleagues investigated FK 778, a drug structurally similar to the active metabolite of leflunomide (A77 1726), and reported that the drug inhibited protein tyrosine phosphorylation and de novo pyrimidine biosynthesis [31]. Studies have been reported demonstrating the efficacy of leflunomide against CMV [32,33]. John and colleagues administered leflunomide to four renal transplant recipients with symptomatic CMV disease. They utilized a loading dose of $100 \mathrm{mg}$ daily for 3 days followed by $20 \mathrm{mg}$ daily for three months [33]. After median treatment duration of a month, all patients had an undetectable viral load with improvement in their symptoms. Leflunomide in combination with foscarnet was reported by Avery and colleagues to have successfully treated a multidrug resistant case of CMV disease in a bone marrow transplant recipient. The target serum level for A77 1726 was set at $60-80 \mu \mathrm{g} / \mathrm{mL}$ [34]. Based on these successful reports, larger studies are warranted to elucidate the efficacy, dosing and long-term safety of leflunomide. 
Citation: Takkar C, Gamilla-Crudo AK, Kadambi PV (2013) Ganciclovir Resistant Cytomegalovirus in Solid Organ Transplant Recipients: An Update. J Transplant Technol Res S6: 001. doi:10.4172/2161-0991.S6-001

\section{Conclusion}

GCV resistant CMV is associated with significant morbidity and poses diagnostic and therapeutic challenges in SOT recipients. Definitive diagnosis can be established by sophisticated lab tests; however, clinical suspicion in the right host is of paramount importance for rapid therapeutic decisions. Given the side effects of conventionally employed second line agents, Leflunomide, due to its immunosuppressive and antiviral activity appears to be an attractive option.

\section{References}

1. Kruger RM, Shannon WD, Arens MQ, Lynch JP, Storch GA, et al. (1999) The impact of ganciclovir-resistant cytomegalovirus infection after lung transplantation. Transplantation 68: 1272-1279.

2. Limaye AP, Raghu G, Koelle DM, Ferrenberg J, Huang ML, et al. (2002) High incidence of ganciclovir-resistant cytomegalovirus infection among lung transplant recipients receiving preemptive therapy. J Infect Dis 185: 20-27.

3. Limaye AP, Corey L, Koelle DM, Davis CL, Boeckh M (2000) Emergence of ganciclovir-resistant cytomegalovirus disease among recipients of solid-organ transplants. Lancet 356: 645-649.

4. Baldanti F, Simoncini L, Sarasini A, Zavattoni M, Grossi P, et al. (1998) Ganciclovir resistance as a result of oral ganciclovir in a heart transplant recipient with multiple human cytomegalovirus strains in blood. Transplantation 66: 324-329.

5. Manso JV, Lambert JL, Oña M, de la Iglesia JL, Iglesias J (1999) Ganciclovir resistance in a heart transplant recipient infected by cytomegalovirus. Int $\mathrm{J}$ Cardiol 71: 97-98.

6. Aitken C, Barrett-Muir W, Raftery M, Breuer J (1999) The clinical significance of ganciclovir resistance in a renal transplant patient. Nephrol Dial Transplant 14: 1050-1051.

7. Bienvenu B, Thervet E, Bedrossian J, Scieux C, Mazeron MC, et al. (2000) Development of cytomegalovirus resistance to ganciclovir after oral maintenance treatment in a renal transplant recipient. Transplantation 69: 182184.

8. Ketteler M, Preuschof L, Mertz A, Stöffler-Meilicke M, Schäfer H, et al. (2000) Fatal cytomegalovirus pneumonia after preemptive antiviral therapy in a renal transplant recipient. Clin Nephrol 54: 418-424.

9. Mendez JC, Sia IG, Tau KR, Espy MJ, Smith TF, et al. (1999) Novel mutation in the CMV UL97 gene associated with resistance to ganciclovir therapy. Transplantation 67: 755-757.

10. Turgeon N, Fishman JA, Doran M, Basgoz N, Tolkoff-Rubin NE, et al. (2000) Prevention of recurrent cytomegalovirus disease in renal and liver transplant recipients: effect of oral ganciclovir. Transpl Infect Dis 2: 2-10.

11. Fishman JA, Doran MT, Volpicelli SA, Cosimi AB, Flood JG, et al. (2000) Dosing of intravenous ganciclovir for the prophylaxis and treatment of cytomegalovirus infection in solid organ transplant recipients. Transplantation 69: 389-394.

12. Rosen HR, Benner KG, Flora KD, Rabkin JM, Orloff SL, etal. (1997) Development of ganciclovir resistance during treatment of primary cytomegalovirus infection after liver transplantation. Transplantation 63: 476-478.

13. Alain S, Honderlick P, Grenet D, Stern M, Vadam C, et al. (1997) Failure of ganciclovir treatment associated with selection of a ganciclovir-resistant cytomegalovirus strain in a lung transplant recipient. Transplantation 63: 15331536.

14. Basgoz N (1999) A 42-year-old lung transplant patient with ganciclovir-resistant cytomegalovirus (CMV) infection. Transpl Infect Dis 1: 218-225.

15. Lurain NS, Ammons HC, Kapell KS, Yeldandi VV, Garrity ER, et al. (1996) Molecular analysis of human cytomegalovirus strains from two lung transplant recipients with the same donor. Transplantation 62: 497-502.
16. Chou S, Waldemer RH, Senters AE, Michels KS, Kemble GW, et al. (2002) Cytomegalovirus UL97 phosphotransferase mutations that affect susceptibility to ganciclovir. J Infect Dis 185: 162-169.

17. Chou S, Guentzel S, Michels KR, Miner RC, Drew WL (1995) Frequency of UL97 phosphotransferase mutations related to ganciclovir resistance in clinical cytomegalovirus isolates. J Infect Dis 172: 239-242.

18. Chou S (1999) Antiviral drug resistance in human cytomegalovirus. Transp Infect Dis 1: 105-114.

19. Erice A (1999) Resistance of human cytomegalovirus to antiviral drugs. Clin Microbiol Rev 12: 286-297.

20. Emery VC, Cope AV, Bowen EF, Gor D, Griffiths PD (1999) The dynamics of human cytomegalovirus replication in vivo. J Exp Med 190: 177-182.

21. Emery VC, Griffiths PD (2000) Prediction of cytomegalovirus load and resistance patterns after antiviral chemotherapy. Proc Natl Acad Sci U S A 97 8039-8044.

22. Limaye AP (2002) Antiviral resistance in cytomegalovirus: an emerging problem in organ transplant recipients. Semin Respir Infect 17: 265-273.

23. Li W, Anwar F, Jesurrun J, Erice A (1999) Cytomegalovirus UL97 and glycoprotein $B(\mathrm{gB})$ sequences in tissues from immunocompromised patients with ganciclovir-resistant virus infection. Scand J Infect Dis 31: 549-553.

24. Wolf DG, Smith IL, Lee DJ, Freeman WR, Flores-Aguilar M, et al. (1995) Mutations in human cytomegalovirus UL97 gene confer clinical resistance to ganciclovir and can be detected directly in patient plasma. J Clin Invest 95 257-263.

25. Gracia-Ahufinger I, Gutiérrez-Aroca J, Cordero E, Vidal E, Cantisán S, et al. (2013) Use of High-Dose Ganciclovir for the Treatment of Cytomegalovirus Replication in Solid Organ Transplant Patients With Ganciclovir ResistanceInducing Mutations. Transplantation 95: 1015-1020.

26. Mylonakis E, Kallas WM, Fishman JA (2002) Combination antiviral therapy for ganciclovir-resistant cytomegalovirus infection in solid-organ transplant recipients. Clin Infect Dis 34: 1337-1341.

27. Biron KK, Harvey RJ, Chamberlain SC, Good SS, Smith AA 3rd, et al. (2002) Potent and selective inhibition of human cytomegalovirus replication by 1263 W94, a benzimidazole L-riboside with a unique mode of action. Antimicrob Agents Chemother 46: 2365-2372.

28. Evers DL, Komazin G, Ptak RG, Shin D, Emmer BT, et al. (2004) Inhibition of human cytomegalovirus replication by benzimidazole nucleosides involves three distinct mechanisms. Antimicrob Agents Chemother 48: 3918-3927.

29. Avery RK, Marty FM, Strasfeld L, Lee I, Arrieta A, et al. (2010) Oral maribavir for treatment of refractory or resistant cytomegalovirus infections in transplant recipients. Transpl Infect Dis 12: 489-496.

30. Waldman WJ, Knight DA, Lurain NS, Miller DM, Sedmak DD, et al. (1999) Novel mechanism of inhibition of cytomegalovirus by the experimenta immunosuppressive agent leflunomide. Transplantation 68: 814-825.

31. Evers DL, Wang X, Huong SM, Andreoni KA, Huang ES (2005) Inhibition of human cytomegalovirus signaling and replication by the immunosuppressant FK778. Antiviral Res 65: 1-12.

32. Zeng $\mathrm{H}$, Waldman WJ, Yin DP, Knight DA, Shen J, et al. (2005) Mechanistic study of malononitrileamide FK778 in cardiac transplantation and CMV infection in rats. Transplantation 79: 17-22.

33. John GT, Manivannan J, Chandy S, Peter S, Jacob CK (2004) Leflunomide therapy for cytomegalovirus disease in renal allograft recepients. Transplantation 77: 1460-1461.

34. Avery RK, Bolwell BJ, Yen-Lieberman B, Lurain N, Waldman WJ, et al. (2004) Use of leflunomide in an allogeneic bone marrow transplant recipient with refractory cytomegalovirus infection. Bone Marrow Transplant 34: 1071-1075.
This article was originally published in a special issue, Solid Organ Transplant: The Pediatric Perspective handled by Editor(s). Dr. Christian Benden, University Hospital Zurich, Switzerland 